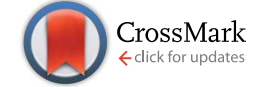

Cite this: RSC Adv., 2017, 7, 4667

Received 12th October 2016 Accepted 11th December 2016

DOI: 10.1039/c6ra25102a

www.rsc.org/advances

\section{In situ synthesis of noble metal nanoparticles on onion-like carbon with enhanced electrochemical and supercapacitor performance $\uparrow$}

\author{
Changda Wang, Chuanqiang Wu, Shuangming Chen, * Qun He, Daobin Liu, \\ Xusheng Zheng, Yasir A. Haleem and Li Song*
}

\begin{abstract}
Ultrafine noble metal nanoparticles decorated on onion-like carbon (OLC) were successfully prepared without any reductant or surfactant. Particularly, the as-prepared Pt-OLC nanocomposite not only exhibited superior electrocatalytic activity and stability for the hydrogen evolution reaction as compared with the commercial $20 \% \mathrm{Pt} / \mathrm{C}$, but also showed enhanced supercapacitor performance with a specific capacitance almost four times larger than that of OLC. Notably, the C-O functional groups on the surface of OLC will increase after the reaction because of the oxidation by $\mathrm{O}_{2}$ and chloroplatinic acid, which is very useful for the reuse of OLC and helpful for the decoration of noble metal nanoparticles on OLC. This work provides a simple and straightforward method for the facile preparation of metal composite nanocatalysts with high catalytic activity.
\end{abstract}

\section{Introduction}

In the past few decades, noble metal nanoparticles with ultrafine size have drawn much attention because of their widespread applications in various fields such as catalysis, fuel cells, gas sensing. ${ }^{1-3}$ However, due to the scarcity and high cost of noble metal materials, it is urgent to reduce their usage. One way to reduce their usage is to incorporate the noble metal nanoparticles onto nano-carbon materials instead of direct use, which shows a great enhancement in specific physical and chemical properties. ${ }^{4,5}$ Based on this, the study of composite of noble metal nanoparticles and nano-carbon materials has become a hot topic in recent years. ${ }^{6,7}$ For all nano-carbon materials, carbon nanotubes and graphene are two promising nano-carbon materials with high specific surface area (SSA). They could supply substrates for the noble metal nanoparticles to grow and anchor. ${ }^{8-10}$ However, the high cost and complicated procedure of the synthesis limit their applications. ${ }^{11,12}$

As a typical nano-carbon material, onion-like carbon (OLC) can be mass-produced by simply annealing of detonation nanodiamond powders. ${ }^{13}$ This kind of OLC usually has a diameter of about $5 \mathrm{~nm}$ and a high SSA larger than $400 \mathrm{~m}^{2} \mathrm{~g}^{-1}{ }^{14}$ Therefore, it is very suitable for noble metal nanoparticles to decorate on and avoid the agglomeration. Furthermore, it is an ideal material for supercapacitors as the unique surface of OLC could be helpful for

National Synchrotron Radiation Laboratory, CAS Center for Excellence in Nanoscience, University of Science and Technology of China, Hefei, Anhui 230029, China.E-mail: csmp@ustc.edu.cn; song2012@ustc.edu.cn

† Electronic supplementary information (ESI) available. See DOI: 10.1039/c6ra25102a ion adsorption and exchange..$^{15}$ Accordingly, the composite of noble metal nanoparticles and OLC may exhibit bifunctional performance both in catalysis and supercapacitor. A number of studies have focused on the synthesis and characterization of OLC-noble metal nanoparticles nanocomposites. ${ }^{16,17}$ Usually, in the previous studies the decoration of noble metal nanoparticles on OLC was achieved with the help of additional reductant and surfactant. ${ }^{18}$ Excellent synthesis method is still lacking.

In this work, we report a simple and novel one-step hydrothermal method for the synthesis of noble metal nanoparticles like Pt, Pd, Au and Ag decorating on OLC. OLC here amazingly acted as both reductant and surfactant in our synthesis. Furthermore, to our best knowledge this is the first time to study the electrochemical property of the OLC-noble metal nanoparticles nanocomposites on hydrogen evolution reaction. Particularly, Pt-OLC nanocomposite not only exhibited excellent electrocatalytic activity and stability for hydrogen evolution reaction, but also showed enhanced stability and specific capacitance for supercapacitor performance.

\section{Results and discussion}

Fig. 1a illustrates the synthesis procedure of Pt nanoparticles decorated OLC sample (Pt-OLC). First of all, nanodiamond (ND) powder was annealed at $1500{ }^{\circ} \mathrm{C}$ and oxidized by mixedacid to get OLC. Then Pt-OLC was obtained by a hydrothermal treatment at $200{ }^{\circ} \mathrm{C}$ without any additional reductant and surfactant. $\mathrm{Pd}, \mathrm{Au}, \mathrm{Ag}$ nanoparticles decorated $\mathrm{OLC}$ samples were also synthesized by the same method. Fig. $1 \mathrm{~b}$ shows the transmission electron microscopy (TEM) image of the Pt-OLC sample, revealing well dispersed Pt nanoparticles on 


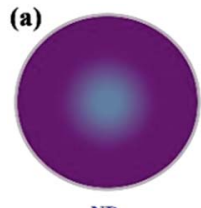

ND

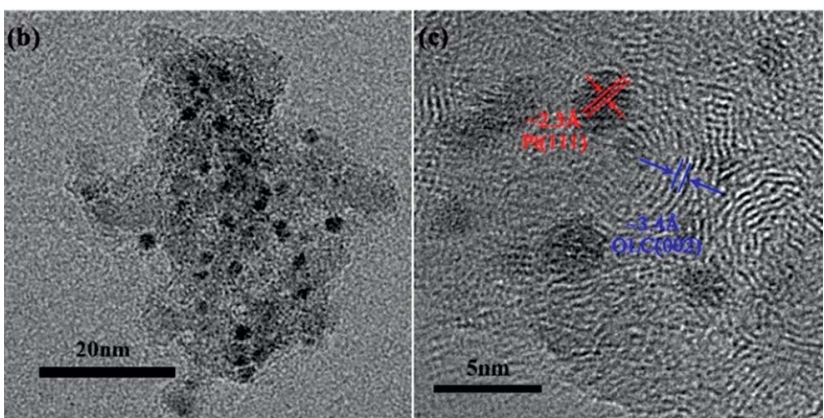

Fig. 1 Schematic illustration of the synthesis process of Pt-OLC sample (a), TEM images of Pt-OLC (b), and its high-resolution TEM (c).

OLC surface. High resolution transmission electron microscopy (HRTEM) image is shown in Fig. 1c. It can be observed that the diameter of OLC particle is about $5 \mathrm{~nm}$ and the decorated $\mathrm{Pt}$ nanoparticles are quasispherical with diameter of 1-2 $\mathrm{nm}$. The interplanar spacing of the graphitic layers of OLC is $\sim 3.4 \AA$, which is consistent with the previous report. ${ }^{19}$ The periodical lattice fringes of the decorated Pt nanoparticles, which can be attributed to (111) plane of face-centered cubic Pt, indicate the high crystallinity of these Pt nanoparticles. It demonstrates that OLC can reduce chloroplatinic acid without any additional reducing agent. Meanwhile, $\mathrm{Pd}, \mathrm{Au}$ and $\mathrm{Ag}$ nanoparticles were also successfully reduced and decorated on OLC (Fig. S1 $\dagger$ ).

To further confirm the decoration of $\mathrm{Pt}$ nanoparticles on OLC and investigate their microstructure, X-ray diffraction (XRD) and $\mathrm{Pt} \mathrm{L}_{3}$-edge X-ray absorption fine spectroscopy (XAFS) measurements were performed as shown in Fig. 2. As we can see
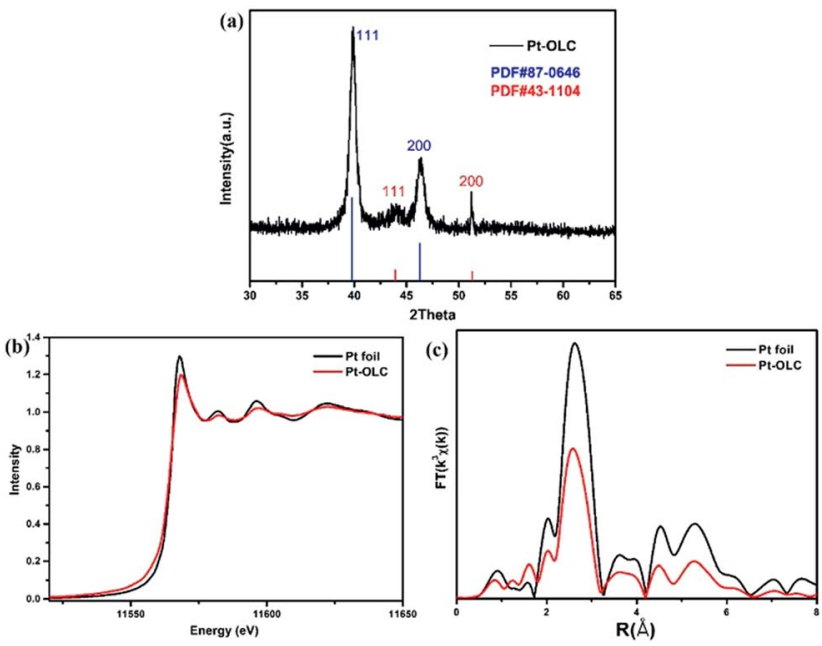

Fig. 2 X-ray diffraction patterns of Pt-OLC (a), the normalized XANES spectra at $P t L_{3}$ edge (b), the Fourier transform spectra from EXAFS of Pt foil and Pt-OLC (c). in Fig. 2a, distinct diffraction peaks of (111) and (200) planes for Pt nanoparticles are observed at $39.80^{\circ}$ and $46.28^{\circ}$ (PDF\#870646, blue). Meanwhile, the other two diffraction peaks at $43.92^{\circ}$ and $51.28^{\circ}$ can be attributed to the (111) and (200) planes of OLC (PDF\#43-1104, red). The high peak intensity of Pt indicates the excellent reduction power of OLC for chloroplatinic acid. The XRD diffraction patterns of Pd-OLC, Au-OLC, Ag-OLC are shown in Fig. S2. $\dagger$

The normalized $\mathrm{Pt} \mathrm{L}_{3}$-edge XANES spectra of Pt foil and PtOLC are compared in Fig. 2b. It can be observed that Pt-OLC has a less intense white line peak compared to Pt foil. As the Pt $\mathrm{L}_{3}$-edge white line probes the electronic transitions from $2 \mathrm{p}_{3 / 2}$ to $5 \mathrm{~d}$ unoccupied states, the decrease intensity of the white line peak suggests that there are more $5 \mathrm{~d}$ electrons for Pt nanoparticles on OLC than Pt foil. It can be attributed to the size effect of nanoparticles, for which the $5 \mathrm{~d}$ electron count of Pt foil is less than that of $\mathrm{Pt}$ nanoparticles because of the $\mathrm{s}-\mathrm{p}-$ $\mathrm{d}$ hybridization. ${ }^{20}$ And that also means there was no oxidation for these Pt nanoparticles decorated on OLC. The corresponding Fourier-transformed EXAFS spectra are shown in Fig. 2c. It can be noted that Pt-OLC has a lower intensity coordination peak than Pt foil at about 2.6 $\AA$, which is also due to the size effect. The best fitting parameters are summarized in Table $\mathrm{S} 1 \dagger$ and the comparison of experimental and fitting curves of PtOLC is shown in Fig. S3.† It can be seen in Table $\mathrm{S} 1 \dagger$ that the coordination number of Pt-Pt in Pt-OLC is 6.6. According to the previous report, ${ }^{21}$ the particle size can be estimated to be around $1 \mathrm{~nm}$, consistent with the above TEM observations.

As there is no additional reductant, it is rationally deduced that the decorated Pt nanoparticles on OLC is attributed to the redox reaction between OLC and chloroplatinic acid, which is similar reaction mechanism as in between Pd nanoparticles and GO proposed by Chen et al. ${ }^{22}$ Thus, the oxygenated functional groups on the OLC surface may play an important role in the formation of Pt nanoparticles. In order to verify the reduction mechanism, we performed the FTIR and XPS spectra measurement.

As we can see in the FTIR spectra of OLC and Pt-OLC in Fig. $\mathrm{S} 4, \dagger$ the peak intensity of $\mathrm{C}=\mathrm{O}$ vibration at wavenumber of $1732 \mathrm{~cm}^{-1}$ decreases clearly after the decoration of Pt nanoparticles. However the $\mathrm{O}-\mathrm{H}$ vibration at $1627 \mathrm{~cm}^{-1}$ which should decrease after the reduction of chloroplatinic acid exhibits no obvious difference. ${ }^{23,24}$ The high-resolution C 1s XPS spectra of OLC and Pt-OLC in Fig. 3 show us more detailed information. The $\mathrm{C}$ 1s core level region can be deconvolved as three peaks at around $284.7 \mathrm{eV}, 286.0 \mathrm{eV}$ and $288.0 \mathrm{eV}$, which are attributed to $\mathrm{C}-\mathrm{C}, \mathrm{C}-\mathrm{O}$ and $\mathrm{C}=\mathrm{O}$ respectively. ${ }^{25}$ Comparing
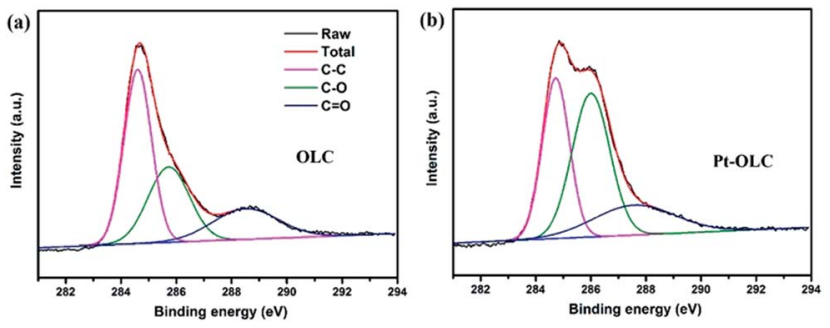

Fig. 3 The high-resolution C 1s XPS spectra of OLC (a), Pt-OLC (b). 
Fig. $3 \mathrm{a}$ with $3 \mathrm{~b}$, it is very intriguing that the relative intensity of $\mathrm{C}-\mathrm{O}$ peak increases obviously after the reaction. This anomalous phenomenon can be assigned to two probable reasons: the oxygen in the water and the chloroplatinic acid, as both of them have strong oxidation ability that can result in the increase of the oxygen-containing functional groups in OLC. To gain an in-depth understanding of the reduction mechanism, two controlling experiments were performed. Ar-OLC samples was synthesized by hydrothermal treatment of OLC solution at $200{ }^{\circ} \mathrm{C}$ with the oxygen removing by argon whereas $\mathrm{O}_{2}-\mathrm{OLC}$ was synthesized by the same way without removing the oxygen in the solution. Fig. $55 \dagger$ displays the XPS results of these two samples. It can be found that the intensity of $\mathrm{C}-\mathrm{O}$ peak for $\mathrm{Ar}-\mathrm{OLC}$ decreases obviously compared with that for $\mathrm{O}_{2}-\mathrm{OLC}$, suggesting that the oxygen in water can oxidize OLC at $200{ }^{\circ} \mathrm{C}$. However, the intensity of $\mathrm{C}-\mathrm{O}$ peak for $\mathrm{O}_{2}-\mathrm{OLC}$ is still weaker than that for Pt-OLC, indicating that chloroplatinic acid can also oxidize OLC under $200{ }^{\circ} \mathrm{C}$. The detailed XPS deconvoluted results are summarized in Table S2.† Therefore, OLC here can not only reduce chloroplatinic acid, but also increase the number of $\mathrm{C}-\mathrm{O}$ functional groups in the surface. It is very useful for the recycling of OLC and helpful for the decoration of noble metal nanoparticles on OLC.

The electrocatalytic activities for HER of the OLC and Pt-OLC samples were examined by electrochemical measurements in $0.5 \mathrm{M} \mathrm{H}_{2} \mathrm{SO}_{4}$, with commercial $20 \% \mathrm{Pt} / \mathrm{C}$ as control samples, results shown in Fig. 4 and Table 1. It can be seen in Fig. $4 a$ that pure OLC exhibits negligible HER activity. On the contrary, PtOLC sample shows very excellent property with an onset potential of $48.6 \mathrm{mV}$ ( $v s$. RHE). It is at the same level compared with the commercial $20 \% \mathrm{Pt} / \mathrm{C}$ whose onset potential is $33.0 \mathrm{mV}$. As comparison, it can be found that the HER performance of Pt-OLC is obviously better than other samples decorated by $\mathrm{Pd}$, Au and $\mathrm{Ag}$ (Fig. S6a $\dagger$ ).

The Tafel plots derived from the polarization are shown in Fig. 4b. Tafel slopes fitted by Tafel equation can be used to reveal the inherent reaction processes of HER. ${ }^{26}$ For Pt-OLC and
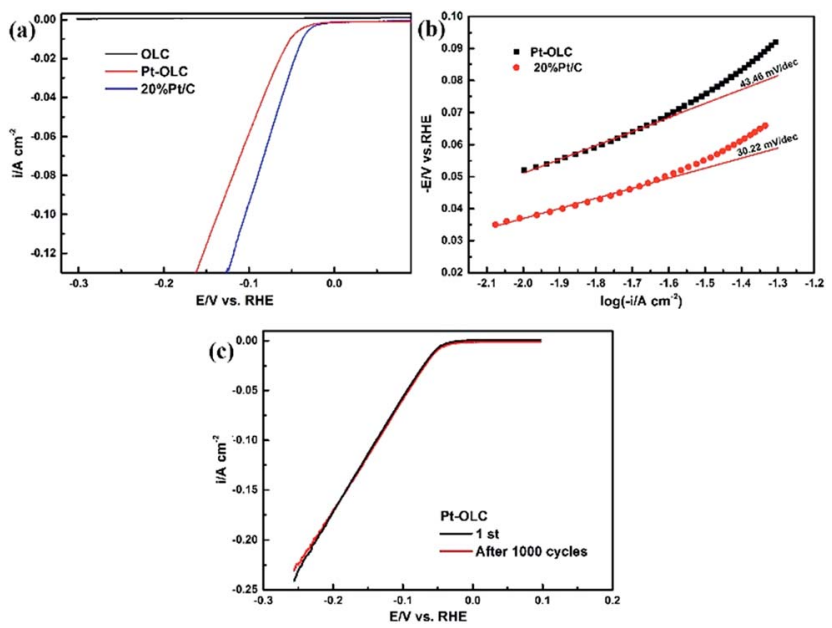

Fig. 4 (a) Polarization curves for $\mathrm{HER}$ in $0.5 \mathrm{M} \mathrm{H}_{2} \mathrm{SO}_{4}$ of pristine OLC, $\mathrm{OLC} / \mathrm{Pt}$ and $20 \% \mathrm{Pt} / \mathrm{C}$ at the scan rate of $5 \mathrm{mV} \mathrm{s}^{-1}$. (b) Corresponding Tafel plots derived from (a). (c) Cycle stability of Pt-OLC sample at a sweep rate of $50 \mathrm{mV} \mathrm{s}^{-1}$.
Table 1 The comparison of Pt-OLC and 20\% Pt/C electrodes on Tafel slop (b), onset potential and the over potential at $10 \mathrm{~mA} \mathrm{~cm}^{-2}\left(\eta_{10}\right)$

\begin{tabular}{llll}
\hline Electrodes & $b / \mathrm{mV} \mathrm{dec}^{-1}$ & Onset potential $/ \mathrm{mV}$ & $\eta_{10} / \mathrm{mV}$ \\
\hline Pt-OLC & 43.46 & 48.6 & 51.04 \\
$20 \% \mathrm{Pt} / \mathrm{C}$ & 30.22 & 33.0 & 37.0 \\
\hline
\end{tabular}

$20 \% \mathrm{Pt} / \mathrm{C}$ samples, the slops of $43.46 \mathrm{mV} \mathrm{dec}^{-1}$ and $30.22 \mathrm{mV}$ $\mathrm{dec}^{-1}$ and the over potentials of $51.04 \mathrm{mV}$ and $37 \mathrm{mV}$ at $10 \mathrm{~mA}$ $\mathrm{cm}^{-2}$ are determined respectively. The Tafel slop of Pt-OLC is much smaller than that of Pd-OLC, Au-OLC and Ag-OLC with the slop of $162.97 \mathrm{mV} \mathrm{dec}{ }^{-1}, 146.86 \mathrm{mV} \mathrm{dec}^{-1}$ and $140.70 \mathrm{mV}$ $\mathrm{dec}^{-1}$ (Fig. S6b $\dagger$ ), demonstrating the superior HER kinetics of Pt-OLC. Moreover, almost the same current density can be obtained after 1000 cycles at a sweep rate of $50 \mathrm{mV} \mathrm{s}^{-1}$ in Fig. 4c, indicating the perfect stability of Pt-OLC sample.

In addition to the HER property, the supercapacitor performance of these samples was also characterized by using electrochemical workstation. ${ }^{27}$ Fig. 5 a compares the CV behavior of OLC and Pt-OLC at the scan rate of $0.1 \mathrm{~V} \mathrm{~s}^{-1}$. The nearly rectangular shape which is typical for double layer capacitors suggests better capacitive behavior for Pt-OLC electrode than OLC electrode. Galvanostatic charge/discharge curves of OLC and Pt-OLC at $1 \mathrm{~A} \mathrm{~g}^{-1}$ are shown in Fig. 5b. The calculated specific capacitance of Pt-OLC is $115.2 \mathrm{~F} \mathrm{~g}^{-1}$, which is almost four times larger than that of OLC $\left(32.4 \mathrm{~F} \mathrm{~g}^{-1}\right)$. Meanwhile, PtOLC shows the largest specific capacitance when compared with $\mathrm{Pd}-\mathrm{OLC}, \mathrm{Au}-\mathrm{OLC}$ and Ag-OLC samples with the specific capacitance of $36.8 \mathrm{~F} \mathrm{~g}^{-1}, 67.2 \mathrm{~F} \mathrm{~g}^{-1}$ and $70.6 \mathrm{~F} \mathrm{~g}^{-1}$ respectively (Fig. S7†). The highly improved supercapacitor performance and the nonlinear behavior of charge/discharge curves can be attributed to the presence of oxygen-containing functional groups and Pt nanoparticles on the surface of OLC, which can bring about some pseudocapacitance. ${ }^{28}$ To clearly study the electrochemical property of Pt-OLC, CV measurement was
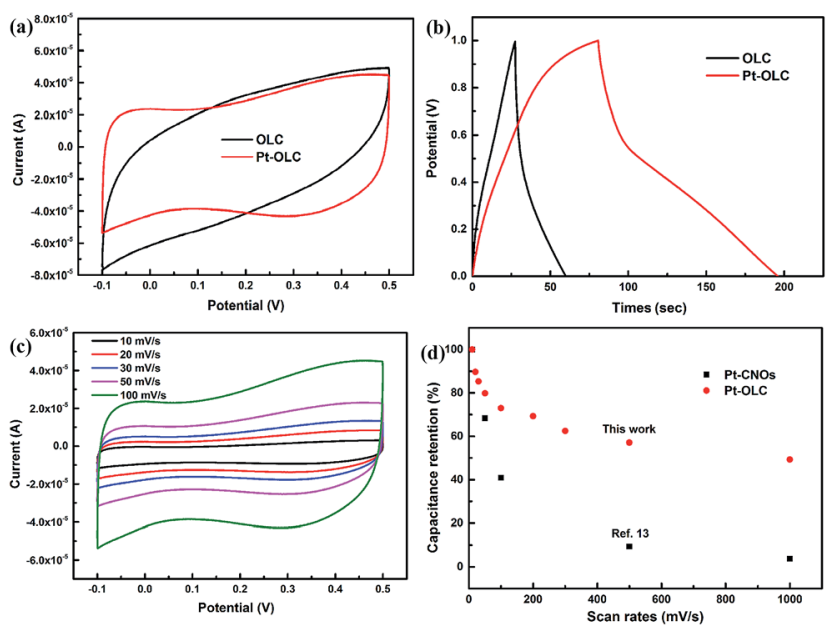

Fig. $5 \mathrm{CV}$ curves of OLC and Pt-OLC at the scan rate of $0.1 \mathrm{~V} \mathrm{~s}^{-1}(\mathrm{a})$, galvanostatic charge/discharge curves of OLC and Pt-OLC at $1 \mathrm{~A} \mathrm{~g}^{-1}$ (b), CV curves of Pt-OLC at different scan rates from $10 \mathrm{mV} \mathrm{s}^{-1}$ to $1000 \mathrm{mV} \mathrm{s}^{-1}$ (c), plot of capacitance retention versus scan rates (d). 
performed. Fig. 5c shows the CV curves of Pt-OLC within -0.1 to $0.5 \mathrm{~V}$ at different scan rate ranging from 10 to $1000 \mathrm{mV} \mathrm{s}^{-1}$. The rectangular shape of $\mathrm{CV}$ curves does not deform at different scan rates, implying the excellent supercapacitor stability of PtOLC. The capacitance retention shown in Fig. 5d indicates the significant stability of Pt-OLC in a wide range of scan rates. It still retains a capacity of about $50 \%$ at the scan rate of $1000 \mathrm{mV}$ $\mathrm{s}^{-1}$ compared with that of $10 \mathrm{mV} \mathrm{s}^{-1}$. The endurance of scan rates is obviously improved compared with the previous report which only retained $3.8 \%$ capacity at $1000 \mathrm{mV} \mathrm{s}^{-1} .^{16}$

\section{Conclusions}

In summary, we have in situ synthesized noble metal nanoparticles decorated on OLC without additional reductant and surfactant. Particularly, the as-prepared Pt-OLC nanocatalyst exhibited very excellent and stable HER performance with the Tafel slop of $43.46 \mathrm{mV} \mathrm{dec}^{-1}$ and pretty small over potential. It also improved the supercapacitor performance with specific capacitance to $115.2 \mathrm{~F} \mathrm{~g}^{-1}$, almost four times larger than that of OLC. Furthermore, we have discovered that the $\mathrm{C}-\mathrm{O}$ functional groups on the surface of OLC have increased after the reaction because of the oxidation by $\mathrm{O}_{2}$ and chloroplatinic acid, which is very helpful for the reuse of OLC and beneficial for decoration of noble metal nanoparticles. This study will open up a simple and straightforward way to synthesize metal composite nanocatalysts with high catalytic activity.

\section{Acknowledgements}

We acknowledge the financial support form National Basic Research Program of China (2014CB848900), the Postdoctoral Science Foundation of China (2015M581990), NSF (U1232131, U1532112, 11375198, 11574280), CUSF (WK2310000053), User with Potential from CAS Hefei Science Center (2015HSC-UP020) and Key Laboratory of Advanced Energy Materials Chemistry (Ministry of Education) Nankai University. L. S. thanks the recruitment program of global experts, the CAS Hundred Talent Program. We also thank the Shanghai synchrotron Radiation Facility (14W1, SSRF), the Beijing Synchrotron Radiation Facility (1W1B and soft-X-ray endstation, BSRF), the Hefei Synchrotron Radiation Facility (MCD, photoemission and Catalysis/Surface Science Endstations, NSRL), and the USTC Center for Micro and Nanoscale Research and Fabrication for help in characterizations and fabrications.

\section{References}

1 J. R. Kitchin, J. K. Norskov, M. A. Barteau and J. G. Chen, J. Chem. Phys., 2004, 120, 10240-10246.

2 M. Murdoch, G. I. Waterhouse, M. A. Nadeem, J. B. Metson, M. A. Keane, R. F. Howe, J. Llorca and H. Idriss, Nat. Chem., 2011, 3, 489-492.

3 X. Luo, W. Xin, C. Yang, D. Yao, Y. Liu, H. Zhang, K. Zhang and B. Yang, Small, 2015, 11, 3583-3590.

4 C. Zhou, Z. Liu, X. Du, D. R. Mitchell, Y. W. Mai, Y. Yan and S. Ringer, Nanoscale Res. Lett., 2012, 7, 165.
5 Y. Li, Y. Li, E. Zhu, T. McLouth, C. Y. Chiu, X. Huang and Y. Huang, J. Am. Chem. Soc., 2012, 134, 12326-12329.

6 G. Wu, C. S. Dai, D. L. Wang, D. Y. Li and N. Li, J. Mater. Chem., 2010, 20, 3059-3068.

7 D. Santiago, G. G. Rodriguez-Calero, A. Palkar, D. BarrazaJimenez, D. H. Galvan, G. Casillas, A. Mayoral, M. JoseYacaman, L. Echegoyen and C. R. Cabrera, Langmuir, 2012, 28, 17202-17210.

8 G. Darabdhara, M. A. Amin, G. A. M. Mersal, E. M. Ahmed, M. R. Das, M. B. Zakaria, V. Malgras, S. M. Alshehri, Y. Yamauchi, S. Szunerits and R. Boukherroub, J. Mater. Chem. A, 2015, 3, 20254-20266.

9 J. M. Liu, H. Meng, J. I. Li, S. J. Liao and J. H. Bu, Fuel Cells, 2007, 7, 402-407.

10 T. Onoe, S. Iwamoto and M. Inoue, Catal. Commun., 2007, 8, 701-706.

11 R. F. Nie, J. H. Wang, L. N. Wang, Y. Qin, P. Chen and Z. Y. Hou, Carbon, 2012, 50, 586-596.

12 L. S. Zhang, X. Q. Liang, W. G. Song and Z. Y. Wu, Phys. Chem. Chem. Phys., 2010, 12, 12055-12059.

13 Y. Gao, Y. S. Zhou, M. Qian, X. N. He, J. Redepenning, P. Goodman, H. M. Li, L. Jiang and Y. F. Lu, Carbon, 2013, 51, 52-58.

14 D. Pech, M. Brunet, H. Durou, P. Huang, V. Mochalin, Y. Gogotsi, P. L. Taberna and P. Simon, Nat. Nanotechnol., 2010, 5, 651-654.

15 Y. Zhai, Y. Dou, D. Zhao, P. F. Fulvio, R. T. Mayes and S. Dai, Adv. Mater., 2011, 23, 4828-4850.

16 S. R. Suryawanshi, V. Kaware, D. Chakravarty, P. S. Walke, M. A. More, K. Joshi, C. S. Rout and D. J. Late, RSC Adv., 2015, 5, 80990-80997.

17 Y. A. Goh, X. Chen, F. M. Yasin, P. K. Eggers, R. A. Boulos, X. Wang, H. T. Chua and C. L. Raston, Chem. Commun., 2013, 49, 5171-5173.

18 H. B. Lu, P. K. Eggers, C. T. Gibson, X. F. Duan, R. N. Lamb, C. L. Raston and H. T. Chua, New J. Chem., 2015, 39, 915-920.

19 W. Gu, N. Peters and G. Yushin, Carbon, 2013, 53, 292-301.

20 Z. Bayindir, P. N. Duchesne, S. C. Cook, M. A. MacDonald and P. Zhang, J. Chem. Phys., 2009, 131, 244716.

21 S. Calvin, M. M. Miller, R. Goswami, S. F. Cheng, S. P. Mulvaney, L. J. Whitman and V. G. Harris, J. Appl. Phys., 2003, 94, 778.

22 X. Chen, G. Wu, J. Chen, X. Chen, Z. Xie and X. Wang, J. Am. Chem. Soc., 2011, 133, 3693-3695.

23 Y. Liu, R. L. Vander Wal and V. N. Khabashesku, Chem. Mater., 2007, 19, 778-786.

24 G. H. Ahmed, R. B. Laino, J. A. Calzon and M. E. Garcia, Beilstein J. Nanotechnol., 2016, 7, 758-766.

25 J. Yang, H. Sun, H. Liang, H. Ji, L. Song, C. Gao and H. Xu, Adv. Mater., 2016, 28, 4606-4613.

26 M. Jahan, Z. L. Liu and K. P. Loh, Adv. Funct. Mater., 2013, 23, 5363-5372.

27 C. Wang, D. Liu, S. Chen, Y. Sang, Y. A. Haleem, C. Wu, W. Xu, Q. Fang, M. Habib, J. Cao, Z. Niu, P. M. Ajayan and L. Song, Small, 2016, 12, 5684-5691.

28 R. Raccichini, A. Varzi, S. Passerini and B. Scrosati, Nat. Mater., 2015, 14, 271-279. 